\title{
Can perineural invasion detected in prostate needle biopsy specimens predict surgical margin positivity in D'Amico low risk patients?
}

\author{
Ozgur Haki Yuksel $^{1}$, Ahmet Urkmez ${ }^{2}$, Ayhan Verit ${ }^{1}$ \\ ${ }^{1}$ Department of Urology, Fatih Sultan Mehmet Research \& Training Hospital, Istanbul, Turkey; \\ ${ }^{2}$ Haydarpasa Numune Research and Training Hospital, Dept. of Urology, Istanbul, Turkey.
}

\begin{abstract}
Summary Objectives: In this study, our aim was to estimate the value of perineural invasion $(\mathrm{PNI})$ in prostate needle biopsy (PNB) specimens in the prediction of surgical margin positivity (SMP) and its prognostic significance (upgrade Gleason Score) in patients who had undergone radical retropubic prostatectomy (RRP) with low risk prostate cancer according to D'Amico risk assessment. Materials and Methods: We retrospectively analyzed the data of 65 patients who were diagnosed as clinical stage T1c prostate cancer $(P C)$ and underwent RRP between January 2010 and June 2013. Pathological specimens of PNB and RRP were separately examined for the parameters of PNI, vascular invasion (VI), Gleason Score (GS) and SMP.

Results: The patients' mean age was $63.65 \pm 4.93$ (range 47 75) years. PNI in PNB specimens were identified in 12 of 65 patients and 11 of 12 patients showed SMP on RRP specimens. While 53 of 65 patients had not PNI on PNB, only 11 of them demonstrated SMP on RRP specimens. SMP was 30.64-fold more frequently encountered in PNB specimens obtained from PNI-positive patients relative to PNI-negative patients. In our study, PNI detected in PNB specimens was statistically significantly associated with SMP on RRP specimens $(P=0.0001)$. Conclusion: It is well known that higher PSA values and GS were independent predictors of SMP in clinically localized prostate cancer (CLPC). We think that PNI in PNB specimens may be a useful prognostic factor for predicting SMP in cases with CLPC.
\end{abstract}

KEY WORDS: Prostate needle biopsy; Prostate adenocarcinoma; Perineural invasion; Lymphovascular invasion; Positive surgical margins.

Submitted 22 October 2015; Accepted 20 January 2016

\section{INTRODUCTION}

Prostate cancer (PC) is one of the most frequently seen fatal malignancies. Detection of the disease at an early stage can ensure complete cure, however uncertainties about actual clinical stage and preoperative prognosis are still prevalent. PC is diagnosed and staged based on histopathological examination of prostate needle biopsy (PNB) specimens or tissue samples obtained from transurethral prostatic resection (TUR-P). Stage of the tumor contained in the radical retropubic prostatectomy
(RRP) specimens is an important marker in the prediction of locally advanced tumor or those with metastatic potential. Besides, tumor stage detected in PNB and RRP specimens is expected to be same. In patients with T1/T2 prostate cancer treated with RRP, biochemical recurrencefree rates were reported as nearly 80 and $60 \%$ within 5 and 10 years of follow-up, respectively (1). Post-RRP recurrence has been most frequently reported within the first postoperative year, which is possibly associated with clinical understaging of the tumors (2). Although a consensus exists proposing surgical margin positivity (SMP) as a prognostic marker, which significantly increases the probability of treatment failure, a standard treatment method specified for these patients is lacking. Besides it is apparent that clinical progression is not observed in all patients (3). PSA levels, Gleason scores (GS), pathological stage, prostate volume (in various studies prostate volume less than 40 gr has been considered as a risk factor for prostate cancer), body mass index (BMI), tumor volume occupying more than $10 \%$ of the prostate, capsular and perineural invasion (PNI) have been defined as basic risk factors for predicting SMP (4). In this study, our aim was to estimate the value of PNI in PNB specimens in the prediction of SMP and prognostic significance (upgrade GS) in patients who had undergone RRP with low risk PC according to D'Amico risk assessment.

\section{MATERIAL AND METHODS}

We retrospectively analyzed data of 65 patients who were diagnosed as clinical stage Tlc PC and underwent RRP between January 2010 and June 2013. All patients underwent 12 quadrant prostate biopsies. Patients, who were diagnosed as low risk PC according to the D'Amico classification, were included in the study. Pathological specimens of PNB and RRP were separately examined for the parameters of PNI, vascular invasion (VI), GS and SMP. All specimens were prospectively processed according to the Stanford protocol (3 $\mathrm{mm}$ ).

Statistical analysis

For statistical evaluation of study data, IBM SPSS Statistics 22 program was used. Compliance with the normal distri- 
bution of parameters was evaluated by Shapiro- Wilks test. In the evaluation of study data, descriptive statistical methods (means, standard deviation, median, frequencies, ratio and minimum, maximum) were used. For the comparison of quantitative data and pairwise intergroup comparisons of variables without normal distribution MannWhitney U test was used. In addition to descriptive statistical methods (means, standard deviation, frequency) for the comparison of qualitative data Fisher's Exact test, chisquare test and Mc Nemar test were used. Statistical significance was evaluated at $\mathrm{p}<0.01$, and $\mathrm{p}<0.05$.

\section{RESULTS}

Patients' mean age was $63.65 \pm 4.93$ (range 47-75) years. Patients' PSA values and prostate volumes ranged respectively between $4-10 \mathrm{ng} / \mathrm{ml}(7.35 \pm 1.96)$ and $18-120 \mathrm{ml}$ (mean: 45,29 $\pm 18,97 \mathrm{ml}$ ). Mean preoperative and postoperative GS of the patients were $5.80 \pm 0.4$ (range: $5-6$ ) and $6.27 \pm 0.80$ (range: $5-9$ ), respectively (Table 1 ).

Increase in postoperative GS of the patients was seen in $23(35.4 \%)$ cases. A statistically significant difference did not exist between incidence rates of increased Gleason scores and presence of preoperative PNI ( $p>0.05)$. Increases in Gleason scores were detected in 38.5, and $34.6 \%$ of the cases with and without preoperative perineural invasion, respectively (Table 2).

Preoperatively PNI was detected in $16.9 \%(\mathrm{n}=11)$ of $25(38.5 \%)$ cases who postoperatively revealed surgical margin positivity, while the remaining cases demonstrated surgical margin negativity. Therefore sensitivity (44.00\%), specificity (97.50\%), accuracy (91.67\%), positive $(73.58 \%)$ and negative (76.92\%) cut-off values of the test were determined as indicated within respective parentheses. SMP was 30.64-fold more frequently encountered in PNB specimens obtained from PNI-positive patients relative to PNI-negative patients [OR: 30.643 (95\% CI: 3.619-259.473)] (Table 3).

\section{Discussion}

In a long-term multiple center study, SMP following RRP has been demonstrated in 10-38\% of the cases (5). Our estimate (38.5\%) appears to be above upper limit of PSM incidence reported in the literature. None of the nomograms predictive of surgical margin have been validated up to now. In a recent study, one of the most important parameters predicting SMP have been indicated as angiolymphatic invasion and GS (6). Efforts aiming at development of an ideal algorithm encompassing preoperative clinical criteria and biopsy results so as to predict SMP are still continuing. Although some authors have asserted that the detection of PNI in needle biopsy specimens might increase risk of extra- prostatic extension, this issue is still debatable. In their systematic review, Harnden et al. (7) investigated the importance of the detection of PNI in cases with postoperative and postradiotherapeutic recurrences and demonstrated its significant prognostic value, especially in patient subgroups defined based on serum PSA levels and GS. They also indicated that patients whose biopsy specimens had evidence of PNI were not suitable for watchful waiting and
Table 1.

Distribution of characteristic features of the patients.

\begin{tabular}{|lcc|}
\hline & Min-max & Mean \pm SD \\
\hline Age & $47-75$ & $63.65 \pm 4.93$ \\
\hline PSA $(\mathrm{ng} / \mathrm{ml})$ & $4.00-10$ & $7.35 \pm 1,96$ \\
\hline Prostate volume (ml) & $18-120$ & $45,29 \pm 18,97$ \\
\hline Preop Gleason score & $5-6$ & $5.80 \pm 0.4$ \\
\hline Postop Gleason score & $5-9$ & $6.26 \pm 0.80$ \\
\hline Prostatic involvement (\%) & $0.30-50.00$ & $13.35 \pm 12.40$ \\
\hline Preop PNI & $\mathbf{N}$ & $\%$ \\
\hline Postop PNI & 12 & 18.5 \\
\hline Preop LVI & 37 & 56.9 \\
\hline Postop LVI & 1 & 1.5 \\
\hline VSI & 17 & 26.2 \\
\hline Surgical margin positivity & 25 & 7.7 \\
\hline $\begin{array}{l}\text { PVol: Prostate volume; PNI: perineural invasion; LVI: lymphovascular invasion } \\
\text { VSl: vesiculo-seminal invasion. }\end{array}$ & \\
\hline
\end{tabular}

\section{Table 2.}

Evaluation of surgical margin positivity and increases in Gleason scores relative to the preoperative presence of perineural invasion.

\begin{tabular}{|c|c|c|c|}
\hline & \multicolumn{2}{|c|}{ Preop PNI } & \\
\hline & $\begin{array}{c}\text { Yes } \\
\text { n (\%) }\end{array}$ & $\begin{array}{c}\text { No } \\
\text { n (\%) }\end{array}$ & \\
\hline Surgical margin positivity & $11(91.6)$ & $14(26.4)$ & $0.001 * *$ \\
\hline Increase in Gleason score & $5(41.6)$ & 18 (33.9) & 1.000 \\
\hline
\end{tabular}

Table 3.

Assessments of preoperative PNI based on the results of surgical margin invasion.

\begin{tabular}{|lc|cc|cc|cc|}
\hline \multirow{2}{*}{} & \multicolumn{8}{|c|}{ Surgical margin invasion } \\
\cline { 3 - 9 } & & \multicolumn{2}{|c|}{ Present } & \multicolumn{2}{|c|}{ Absent } & \multicolumn{2}{c|}{ Total } \\
& & $\mathbf{n}$ & $\%$ & $\mathbf{n}$ & $\%$ & $\mathbf{n}$ & $\%$ \\
\hline Preop & Yes & 11 & 16.9 & 1 & 1.5 & $\mathbf{1 2}$ & $\mathbf{1 8 . 5}$ \\
\cline { 3 - 9 } PNI & No & 14 & 21.5 & 39 & 60.0 & $\mathbf{5 3}$ & $\mathbf{8 1 . 5}$ \\
\cline { 3 - 8 } & Total & $\mathbf{2 5}$ & $\mathbf{3 8 . 5}$ & $\mathbf{4 0}$ & $\mathbf{6 1 . 5}$ & $\mathbf{6 5}$ & $\mathbf{1 0 0}$ \\
\hline
\end{tabular}

they required early therapeutic intervention. In more than $67 \%$ of the studies where external radiotherapy was applied (excluded brachytherapy) prognostic value of PNI has been demonstrated.

In the year 2007, Passavanti et al. detected 53\% PNI positivity in RRP specimens of 94 patients, and demonstrated PNI positivity in PNB samples of only $45 \%$ of these cases. In our study, we observed PNI-positivity in $18.5 \%(n=12)$ of biopsies, but $56.9 \%(n=37)$ of radical prostatectomy specimens. Passavanti et al. analyzed patients with serum PSA levels between 4 and $20 \mathrm{ng} / \mathrm{ml}$ in their study and confirmed the correlation between PNI and higher GS as was also proved in our study. As 
a result of their study, Passavanti et al. revealed significance of PNI-positivity detected in PNB specimens for the treatment plan, while PNI positivity found in RRP specimens was indicated to be meaningful for biological behaviour and progression of the tumor (8). However in our study, which took in consideration PSA interval between 4-10 ng/ml, a statistical correlation between PNI and GS upgrade was not detected. PNI in PNB was considered as an important criterion in the decisionmaking of active surveillance in several studies. Still, in these studies, PNI was associated with higher tumor volume, which did not indicate a poor prognosis (9-11). In an investigation performed by Walsh and Epstein in 1993, the authors reported that knowledge about the presence and extent of PNI in needle biopsy specimens might provide information about capsular penetration and aid in the decision-making process for nerve-sparing surgery (12). In another prognostic study, PNI was found to be correlated independently with adverse histopathological characteristics and worse survival outcomes after RRP (13).

Since higher percentage of cases with extraprostatic tumoral extension associated with PNI have been reported in the literature, some authors have advocated routine resection of neurovascular bundle at the PNI-positive side so as to achieve an ipsilateral surgical margin negativity. However Cannon et al. conducted a multivariate analysis on 425 patients in the year 2005 and reported presence of a weak correlation between PNI-positivity and SMP contrary to a significant correlation between PNI-positivity and organ-confined disease. They indicated that though PNI-positivity highly predicts extracapsular extension, bilateral nerve-sparing surgery is not required in these patients (14).

In a multivariate analysis of preoperative PSA levels, GS, digital rectal examination, tumor volume and PNI, PNI could predict extraprostatic invasion in nearly $10 \%$ of the cases (15). However in another study, any correlation between PNI and GS estimated based on histopathological examination of RRP specimens, extraprostatic extension, SMP, LVI and upgrading of the tumor was not detected (16). In a study similar to ours, PNI was indicated as an effective factor on SMP and PSA recurrence during 5 years of follow-up in patients who had undergone RRP with the indication of localized prostate cancer (17). This study documents that longterm prostate cancer outcomes are best estimated with a combination of GS, detection of PNI and Ki-67 expression. Given its low cost, rapid assessment and strong predictive power, we believe that combining presence of Ki-67 expression, PNI and estimated GS based on histopathological examination of biopsy specimens should be considered as a standard by which all new biomarkers must be compared with before introducing them into clinical practice (18).

We know the importance of estimated GS of PNB specimens, preoperative PSA values and PNI in the prediction of postoperative clinical course. Though we have observed that higher PSA and GS predicted SMP at a large extent, we have also seen SMP in patients with relatively lower GS and PSA values independent of histopathological characteristics of the surgical speci- men. Uncertainties exist about criteria on which clinicians should base their therapeutic decisions. We think that criteria of PNI detected in PNB specimens can explain and predict this phenomenon. Similarly, as described above, even though relevant data are debatable, it has been asserted that determinable characteristic of PNI may predict SMP and even in some studies prognostic value of PNI has been specified. We think that questioning the place of PNI in the prediction of SMP is the most accurate approach in patients categorized in the lower risk group according to D'Amico classification, In parallel with this assumption, in our study, we observed that PNI detected in histopathological examination of the biopsy specimens could predict SMP independent of PSA and GS. Therefore, we conceive that inclusion of histopathologically detected PNI in preoperative nomograms should be debatable.

The limitations of our study were that it was retrospective one with limited study group and had not a prognostic predictive design. Prospective studies are needed to have data on disease-free survival in patients with PNI at biopsy who underwent to prostatectomy, and eventually understand if PNI can be a decisive factor in the choice of not radical treatment for the prostate cancer detected at biopsy.

\section{REFERENCES}

1. Yossepowitch O, Bjartell A, Eastham JA, et al. Positive surgical margins in radical prostatectomy: outlining the problem and its long-term consequences. Eur Urol. 2009; 55:87-99.

2. Eastham JA, Scardino PT. Radical prostatectomy; in Walsh PC (ed). Campbell's Urology. Philadelphia, Elsevier, 2005: 3080-102.

3. Sofer M, Hamilton-Nelson KL, Civantos F, et al. Positive surgical margins after radical retropubic prostatectomy: the influence of site and number on progression. J Urol. 2002; 167:2453-6.

4. Ko J, Falzarano SM, Walker E, et al. Prostate cancer patients older than 70 years treated by radical prostatectomy have higher biochemical recurrence rate than their matched younger counterpart. Prostate. 2013; 73:897-903.

5. Schiavina R, Borghesi $M$, Dababneh $H$, et al. Survival, Continence and Potency (SCP) recovery after radical retropubic prostatectomy: A long-term combined evaluation of surgical outcomes. Eur J Surg Oncol. 2014; 40:1716-23.

6. La Roca RL, Cunha IW, Bezerra SM, et al. Radical prostatectomy and positive surgical margins: Relationship with prostate cancer outcome. Int Braz J Urol. 2014; 40:306-15.

7. Harnden P, Shelley MD. The prognostic significance of perineural invasion in prostatic cancer biopsies: A systematic review. Cancer. 2007; 109:13-24.

8. Passavanti G, Pizzuti V. Perineural invasion in prostatic carcinoma treated with radical prostatectomy: The role of TR systematic biopsy. Arch Ital Urol Androl. 2007; 79:23-5.

9. Trpkov C, Yilmaz A, Trpkov K. Perineural invasion in prostate cancer patients who are potential candidates for active surveillance: Validation study. Urology. 2014; 84:149-52.

10. Cohn JA, Dangle PP, Wang CE, et al. The prognostic significance of perineural invasion and race in men considering active surveillance. BJU Int. 2014; 114:75-80. 
11. Gorin MA, Chalfin HJ, Epstein JI, et al. Predicting the risk of non-organ-confined prostate cancer when perineural invasion is found on biopsy. Urology. 2014; 83:1117-21.

12. Bastacky SI, Walsh PC, Epstein JI. Relationship between perineural tumor invasion on needle biopsy and radical prostatectomy capsular penetration in clinical stage $B$ adenocarcinoma of the prostate. Am J SurgPathol. 1993; 17:336-41.

13. DeLancey JO, Wood DP Jr, He C, et al. Evidence of perineural invasion on prostate biopsy specimen and survival after radical prostatectomy. Urology. 2013; 81:354-7.

14. Cannon GM Jr, Pound CR. Perineural invasion in prostate cancer biopsies is not associated with higher rates of positive surgical margins. Prostate. 2005; 63:336-40.

15. Tsuzuki T, Hernandez DJ, Aydin H, et al. Prediction of extrapro- static extension in the neurovascular bundle based on prostate needle biopsy pathology, serum prostate specific antigen and digital rectal examination. J Urol. 2005; 173:450-3.

16. Elharram M, Margel D, Finelli A, et al. Perineural invasion on prostate biopsy does not predict adverse pathological outcome. Can J Urol. 2012; 19:6567-72.

17. D'Amico AV, Wu Y, Chen MH, et al. Perineural invasion as a predictor of biochemical outcome following radical prostatectomy for select men with clinically localized prostate cancer. J Urol. 2001; 165:126-9.

18. Tollefson MK, Karnes RJ, Kwon ED, et al. Prostate cancer Ki-67 (MIB-1) expression, perineural invasion, and gleason score as biopsy-based predictors of prostate cancer mortality: the Mayo model. Mayo Clin Proc. 2014; 89:308-18.

\section{Correspondence}

Ozgur Haki Yuksel, MD (Corresponding Author)

ozgurhaki@gmail.com

Ayhan Verit, MD, Prof.

Department of Urology, Fatih Sultan Mehmet Research \& Training Hospital Içerenköy/Atasehir Tr- 34752 Istanbul, Turkey

Ahmet Urkmez, MD

Haydarpasa Numune Research and Training Hospital, Dept. of Urology

Istanbul, Turkey 